\title{
Cytomorphological Characterization in 28 Species of Papilionaceae from Indian Cold Deserts
}

\author{
Dalvir Kaur ${ }^{1}$, Vijay Kumar Singhal ${ }^{2}$ \\ ${ }^{1}$ Assistant Professor, Department of Botany, Patel Memorial National College, Rajpura, Patiala, Punjab, India \\ ${ }^{2}$ Professor, Department of Botany, Punjabi University, Patiala, Punjab, India
}

\begin{abstract}
Present cytomorphological investigation in Papilionaceous members from cold desert region of Kinnaur (India) record the first ever chromosome count in Astragalus malacophyllus ( $n=8)$, A. strobiliferus $(n=8)$, Campylotropis eriocarpa (n=11), Colutea nepalensis $(n=8)$, Indigofera himalayensis $(n=8)$, Trigonella pubescens $(n=8)$, Vicia rigidula $(n=12), V$. tenera $(n=7)$ and in variety nutans of Desmodium elegans $(n=11)$ at world level and in Caragana gerardiana $(n=8)$, Desmodium racemosum $(n=11)$, Medicago falcata $(n=8)$, and Piptanthus nepalensis $(n=9)$ at India level. New or variable chromosome counts have been recorded for four species named Indigofera heterantha, Oxytropis mollis, Trigonella emodi, and Vicia pallida. Based on $x=8$, in Indigofera heterantha existence of intraspecific diploid $(n=8)$ and hexaploid $(n=24)$ cytotypes have been reported. In this species, the diploid and hexaploid individuals can be distingiushed from each other on the basis of vegetative and floral characters. Further meiotic course in majority of species was observed perfectly regular which resulting into high pollen fertility. However, 11 species depicted the phenomenon of cytomixis of inter PMCs chromatin transfer which consequences various irregular meiotic products yielded unstained/sterile and fertile pollen grains of variable sizes.
\end{abstract}

Keywords: chromosome counts, intraspecific polyploidy, cytomixis, pollen grains

\section{Introduction}

The Papilionaceae is the third largest and economically important family of angiosperms [7] with approximately 476 genera and over 13860 species worldwide, which are easily recognized by their legume fruit and their compound and stipulated leaves. The family is well represented throughout temperate and tropical regions of the world [10] ranging in habit from large trees to annual herbaceous plants. The present communication covers the chromosomal counts, detailed male meiotic course and pollen fertility \%age in some members belonging to family Papilionaceae from Kinnaur district of Himachal Pradesh in India (Map 1). Kinnaur is tribal and hilly district with altitude ranging from $1600-6800 \mathrm{~m}$, represents a number of lush green valleys, mountain ranges, snow-clad peaks and cold desert conditions. In spite of harsh climatic conditions, the area is rich in plant diversity which includes plenty of flowering plants of dry alpine and temperate zones. Besides some of the species are confined to specific habitats like rock crevices, dry sandy slopes, among stones, moist open and shaddy slopes and along stream side, river and water channels. Owing to rich plant diversity, Kinnaur district has been well explored for its medicinal and aromatic plants wealth [3] [9]. Presently an attempt has been made to explore the cytomorphological diversity in papilionaceous members from this high altitudinal and cold desert region of northwest Himalayas.

\section{Material and Methods}

\subsection{Collection and Submission of Samples}

For exploration of cytomorphological diversity, study materials were collected by surveying the different localities of Kinnaur district during the months of April to September for five years (2007-2011). Voucher specimens of the cytomorphologically worked out individuals were deposited in the Herbarium, Department of Botany, Punjabi University Patiala (PUN).

\subsection{Cytological Analysis}

Young panicles/floral buds were fixed in freshly prepared Carnoy's fixative (6 ethanol: 3 chloroform: 1 acetic acid v: v: v) for $24 \mathrm{~h}$ and preserved in $70 \%$ alcohol at $4^{\circ} \mathrm{C}$. Anthers were squashed in $1 \%$ acetocarmine and in each case $400-500$ sporads were analyzed. Pollen viability was estimated through stainability tests for which anthers from mature flowers were squashed in glycerol-acetocarmine (1:1) mixture and $1 \%$ aniline blue dye. Well filled pollen grains with fully stained nuclei and cytoplasm were scored as fertile while shrivelled with partially or unstained cytoplasm were counted as sterile.

\subsection{Morphometric Analysis}

The morphological analysis is evaluated by field observations to mark out the different morphovariants by focusing on vegetative (plant height, number of branches per plant, number of leaflets per leaf, size and colour of leaves) and reproductive (number of flowers per infloresnce and colour of flowers) parameters. Stomatal studies were made from the abaxial epidermal peels of the leaves through $\mathrm{KOH}$ treatment. Following expression was used to calculate the stomatal index: $\mathrm{SI}=\mathrm{S} / \mathrm{E}+\mathrm{S} \times 100$

Where, $\mathrm{SI}=$ stomatal index, $\mathrm{S}=$ number of stomata per field, $\mathrm{E}=$ number of epidermal cells per field.

\subsection{Photomicrographs}

Photomicrographs of chromosome counts, meiotic abnormalities, sporads, pollen grains, and stomata were made from the freshly prepared slides using Leica Qwin Digital Imaging System (X290, X900 and X2180) and Nikon Eclipse 80i microscope (X330, X1340 and X3400). 


\section{International Journal of Science and Research (IJSR) \\ ISSN (Online): 2319-7064 \\ Index Copernicus Value (2013): 6.14 | Impact Factor (2015): 6.391}

\section{Results and Discussions}

Present work includes detailed cytomorphological studies on 28 species (41 accessions) under 14 genera of family Papilionaceae from different localities of Kinnaur district between altitudes of $1680-3660 \mathrm{~m}$. Data on species, localities with altitudes, GPS coordinates, accession number (PUN), meiotic chromosome number, ploidy level, PMCs involved in cytomixis (\%age), pollen fertility (\%age) and previous chromosome counts are provided in Table 1 . Some of the interesting findings include-

\subsection{First ever chromosome counts}

All the species of the family have been worked out cytologically for the first time from geographical isolated and cold desert region of Kinnaur. Astragalus malacophyllus ( $\mathrm{n}=8$; Fig. 1), A. strobiliferus (n=8; Fig. 2), Campylotropis eriocarpa ( $\mathrm{n}=11$; Fig. 3), Colutea nepalensis ( $\mathrm{n}=8$; Fig. 4), Indigofera himalayensis $(\mathrm{n}=8$; Fig. 5), Trigonella pubescens $(\mathrm{n}=8$; Fig. 6), Vicia rigidula $(\mathrm{n}=12$; Fig. 7$)$, V. tenera $(\mathrm{n}=7$; Fig. 8) are counted chromosomally for the first time at world level. The variety nutans of Desmodium elegans $(\mathrm{n}=11$; Fig. 9) is also recorded chromosomally for the first time. The study also includes the first ever chromosomal count from India for Caragana gerardiana ( $\mathrm{n}=8$; Fig. 10), Desmodium racemosum (n=11; Fig. 11), Medicago falcata (n=8; Fig. 12), and Piptanthus nepalensis ( $\mathrm{n}=9$; Fig. 13).

\subsection{Additional/variable cytotypes}

On the basis of present chromosome study, new or variable counts have been recorded for four species named Indigofera heterantha, Oxytropis mollis, Trigonella emodi, and Vicia pallida. The diploid ( $2 n=16$; Fig. 17) cytotype in Indigofera heterantha is recorded for the first time from India but already counted from Pakistan [2]. Oxytropis mollis with 2n=16 (Fig. 14) adds a new intraspecific diploid cytotype against already existing tetraploid

Table 1: Information on locality with altitude and GPS Coordinates, accession number/s (PUN*), meiotic chromosome number, ploidy level, PMCs involved in cytomixis (\%age), pollen fertility (\%age), and previous chromosome reports on the cytologically investigated species of Family Papilionaceae.

\begin{tabular}{|c|c|c|c|c|c|c|c|c|c|}
\hline $\begin{array}{l}\text { S. } \\
\text { No. }\end{array}$ & Taxon & $\begin{array}{l}\text { Locality with } \\
\text { altitude }(\mathrm{m})\end{array}$ & $\begin{array}{l}\text { Localities with GPS } \\
\quad \text { Coordinates }\end{array}$ & $\begin{array}{c}\text { Accession } \\
\text { number/s } \\
\left(P U N^{*}\right)\end{array}$ & $\begin{array}{c}\text { Meiotic } \\
\text { chromosome } \\
\text { number }(n)\end{array}$ & $\begin{array}{c}\text { Ploidy } \\
\text { level }\end{array}$ & $\begin{array}{l}\text { PMCs } \\
\text { involved } \\
\quad \text { in } \\
\text { cytomixis } \\
\text { (\%age) }\end{array}$ & \begin{tabular}{|c|} 
Pollen \\
fertility \\
(\%age)
\end{tabular} & $\begin{array}{l}\text { Previous } \\
\text { chromosome } \\
\text { reports** }\end{array}$ \\
\hline 1. & $\begin{array}{l}\text { Astragalus } \\
\text { candolleanus } \\
\text { Royle }\end{array}$ & $\begin{array}{l}\text { Rakchham, } \\
3115\end{array}$ & $31^{\circ} 22^{\prime} 49^{\prime \prime N ~ 78} 22^{\prime} 5^{\prime \prime E}$ & 53678 & 8 & $2 \mathrm{x}$ & - & 100 & $2 n=16$ \\
\hline 2. & $\begin{array}{l}\text { A. } \\
\text { chlorostachys } \\
\text { Lindl. }\end{array}$ & Sangla, 2680 & $31^{\circ} 25^{\prime} 36^{\prime \prime} \mathrm{N} 78^{\circ} 15^{\prime} 51^{\prime \prime E}$ & 50909 & 8 & $2 \mathrm{x}$ & - & 100 & $2 n=16$ \\
\hline 3. & $\begin{array}{l}\text { A. } \\
\text { grahamianus } \\
\text { Royle ex } \\
\text { Benth. }\end{array}$ & $\begin{array}{l}\text { Rakchham, } \\
3115\end{array}$ & $31^{\circ} 22^{\prime} 49^{\prime \prime} \mathrm{N} 78^{\circ} 22^{\prime} 5^{\prime \prime E}$ & 53673 & 8 & $2 x$ & 17.27 & 92 & $2 n=16$ \\
\hline 4. & $\begin{array}{l}\text { A. graveolens } \\
\text { Buch.-Ham. ex } \\
\text { Benth. }\end{array}$ & \begin{tabular}{|l|} 
Ropa, 3000 \\
Ropa, 3000 \\
Sangla, 2680 \\
Rakchham, \\
3115 \\
Karchham, 1900 \\
\end{tabular} & $\begin{array}{l}31^{\circ} 47^{\prime} 48^{\prime \prime} \mathrm{N} 78^{\circ} 25^{\prime} 22^{\prime \prime} \mathrm{E} \\
31^{\circ} 25^{\prime} 36^{\prime \prime} \text { " } 78^{\circ} 15^{\prime} 51^{\prime \prime} \mathrm{E} \\
31^{\circ} 22^{\prime} 49^{\prime \prime N} 78^{\circ} 22^{\prime} 5^{\prime \prime} \mathrm{E} \\
31^{\circ} 29^{\prime} 46^{\prime \prime} \mathrm{N} 78^{\circ} 10^{\prime} 47^{\prime \prime} \mathrm{E}\end{array}$ & $\begin{array}{l}55758 \\
55759 \\
50900 \\
53672 \\
50910\end{array}$ & $\begin{array}{l}8 \\
8 \\
8 \\
8 \\
8\end{array}$ & $\begin{array}{l}2 x \\
2 x \\
2 x \\
2 x \\
2 x\end{array}$ & $\begin{array}{l}17.02- \\
20.55\end{array}$ & $\begin{array}{c}100 \\
100 \\
97 \\
96 \\
100\end{array}$ & $2 n=16$ \\
\hline 5. & $\begin{array}{l}\text { A. } \\
\text { malacophyllus } \\
\text { Benth. ex } \\
\text { Bunge } \\
\end{array}$ & $\begin{array}{l}\text { Rakchham, } \\
3115\end{array}$ & $31^{\circ} 22^{\prime} 49^{\prime \prime} \mathrm{N} 78^{\circ} 22^{\prime} 5^{\prime \prime E}$ & 53676 & 8 & $2 \mathrm{x}$ & - & 100 & \# \\
\hline 6. & $\begin{array}{l}\text { A. rhizanthus } \\
\text { Royle ex } \\
\text { Benth. }\end{array}$ & Chittkul, 3450 & $31^{\circ} 21^{\prime} 7 " \mathrm{~N} 78^{\circ} 26^{\prime} 7^{\prime \prime E}$ & 53677 & 8 & $2 x$ & - & 100 & $2 n=16$ \\
\hline 7. & $\begin{array}{l}\text { A. strobiliferus } \\
\text { Royle } \\
\end{array}$ & Ropa, 3000 & $31^{\circ} 47^{\prime} 48^{\prime \prime} \mathrm{N} 78^{\circ} 25^{\prime} 22^{\prime \prime} \mathrm{E}$ & 53680 & 8 & $2 \mathrm{x}$ & - & 98 & \# \\
\hline 8. & $\begin{array}{l}\text { A. zanskarensis } \\
\text { Benth. ex } \\
\text { Bunge } \\
\end{array}$ & Chittkul, 3450 & $31^{\circ} 21^{\prime} 7^{\prime \prime N ~} 78^{\circ} 26^{\prime} 7^{\prime \prime E}$ & 53682 & 8 & $2 \mathrm{x}$ & - & 100 & $2 n=16$ \\
\hline 9. & $\begin{array}{l}\text { Campylotropis } \\
\text { eriocarpa } \\
\text { Schindler }\end{array}$ & $\begin{array}{l}\text { Bhabanagar, } \\
1900\end{array}$ & $31^{\circ} 33^{\prime} 50^{\prime \prime} \mathrm{N} 77^{\circ} 56^{\prime} 17^{\prime \prime E}$ & 50913 & 11 & $2 \mathrm{x}$ & - & 100 & $\#$ \\
\hline 10. & \begin{tabular}{|l} 
Caragana \\
gerardiana \\
Royle \\
\end{tabular} & Nako, 3660 & $31^{\circ} 52^{\prime} 50^{\prime \prime} \mathrm{N} 78^{\circ} 37^{\prime} 37^{\prime \prime E}$ & 53683 & 8 & $2 \mathrm{x}$ & - & 98 & $2 n=16,18$ \\
\hline 11. & Colutea & Pooh, 2840 & $31^{\circ} 45^{\prime} 47^{\prime \prime} \mathrm{N} 78^{\circ} 35^{\prime} 20^{\prime \prime} \mathrm{E}$ & 53686 & 8 & $2 x$ & - & 100 & $\#$ \\
\hline
\end{tabular}




\section{International Journal of Science and Research (IJSR) \\ ISSN (Online): 2319-7064}

Index Copernicus Value (2013): 6.14 | Impact Factor (2015): 6.391

\begin{tabular}{|c|c|c|c|c|c|c|c|c|c|}
\hline & \begin{tabular}{|l|} 
nepalensis \\
Sims
\end{tabular} & $\begin{array}{l}\text { Khab, } 2800 \\
\text { Moorang, } 2590 \\
\text { Thangi, } 2700\end{array}$ & $\begin{array}{c}31^{\circ} 48^{\prime} 0^{\prime \prime N} 78^{\circ} 38^{\prime} 38^{\prime \prime} \mathrm{E} \\
31^{\circ} 35^{\prime} 18^{\prime \prime N} 78^{\circ} 26^{\prime} 53^{\prime \prime} \mathrm{E} \\
31^{\circ} 33^{\prime} 6^{\prime \prime N} 78^{\circ} 28^{\prime} 57^{\prime \prime} \mathrm{E}\end{array}$ & $\begin{array}{l}53684 \\
53685 \\
53687\end{array}$ & $\begin{array}{l}8 \\
8 \\
8\end{array}$ & $\begin{array}{l}2 \mathrm{x} \\
2 \mathrm{x} \\
2 \mathrm{x}\end{array}$ & & $\begin{array}{l}95 \\
99 \\
98\end{array}$ & \\
\hline 12. & $\begin{array}{l}\text { Desmodium } \\
\text { elegans DC. } \\
(=D . \\
\text { tiliaefolium (D. } \\
\text { Don) G. Don, } \\
=\text { Hedysarum } \\
\text { tiliaefolium D. } \\
\text { Don) } \\
\end{array}$ & $\begin{array}{l}\text { Kalpa, } 2760 \\
\text { Kuppa, } 2650 \\
\text { Sangla, } 2680\end{array}$ & $\begin{array}{l}31^{\circ} 31^{\prime} 55^{\prime \prime} \mathrm{N} 78^{\circ} 15^{\prime} 5^{\prime \prime E} \\
31^{\circ} 25^{\prime} 44^{\prime \prime} \mathrm{N} 78^{\circ} 14^{\prime} 40^{\prime \prime} \mathrm{E} \\
31^{\circ} 25^{\prime} 36^{\prime \prime} \mathrm{N} 78^{\circ} 15^{\prime} 51^{\prime \prime} \mathrm{E}\end{array}$ & $\begin{array}{l}54277 \\
54278 \\
54279\end{array}$ & $\begin{array}{l}11 \\
11 \\
11\end{array}$ & $\begin{array}{l}2 \mathrm{x} \\
2 \mathrm{x} \\
2 \mathrm{x}\end{array}$ & - & $\begin{array}{c}98 \\
99 \\
100\end{array}$ & $2 n=22$ \\
\hline & $\begin{array}{l}\text { D. elegans DC. } \\
\text { var. nutans } \\
\text { (Hook.) H. } \\
\text { Ohashi }\end{array}$ & \begin{tabular}{|l} 
Rakchham, \\
3115 \\
Tapri, 1680 \\
Chaura, 1890 \\
Bhabanagar, \\
1900 \\
Karchham, \\
1900 \\
Karchham, \\
1900 \\
Palingi, 1900 \\
\end{tabular} & $\begin{array}{l}31^{\circ} 22^{\prime} 49^{\prime \prime} \mathrm{N} 78^{\circ} 22^{\prime} 5^{\prime \prime} \mathrm{E} \\
31^{\circ} 31^{\prime} 44^{\prime \prime} \mathrm{N} 78^{\circ} 6^{\prime} 0^{\prime \prime} \mathrm{E} \\
31^{\circ} 33^{\prime} 50^{\prime \prime} \mathrm{N} 77^{\circ} 56^{\prime} 17^{\prime \prime} \mathrm{E} \\
31^{\circ} 33^{\prime} 50^{\prime \prime} \mathrm{N} 77^{\circ} 56^{\prime} 17^{\prime \prime} \mathrm{E} \\
31^{\circ} 29^{\prime} 46^{\prime \prime} \mathrm{N} 78^{\circ} 10^{\prime} 47^{\prime \prime} \mathrm{E} \\
31^{\circ} 29^{\prime} 46^{\prime \prime} \mathrm{N} 78^{\circ} 10^{\prime} 47^{\prime \prime} \mathrm{E} \\
31^{\circ} 33^{\prime} 23^{\prime \prime} \mathrm{N} 77^{\circ} 52^{\prime} 58^{\prime \prime} \mathrm{E}\end{array}$ & $\begin{array}{l}53670 \\
53671 \\
53667 \\
53665 \\
53668 \\
53666 \\
53669\end{array}$ & $\begin{array}{l}11 \\
11 \\
11 \\
11 \\
11 \\
11 \\
11\end{array}$ & $\begin{array}{l}2 \mathrm{x} \\
2 \mathrm{x} \\
2 \mathrm{x} \\
2 \mathrm{x} \\
2 \mathrm{x} \\
2 \mathrm{x} \\
2 \mathrm{x}\end{array}$ & - & $\begin{array}{l}100 \\
100 \\
100 \\
100 \\
100 \\
100 \\
100\end{array}$ & $\#$ \\
\hline 13. & $\begin{array}{l}\text { D. racemosum } \\
\text { (Thunb.) DC. }\end{array}$ & Kalpa, 2760 & $31^{\circ} 31^{\prime} 55^{\prime \prime N} 78^{\circ} 15^{\prime} 5^{\prime \prime E}$ & 53664 & 11 & $2 \mathrm{x}$ & & 100 & $2 n=22$ \\
\hline 14. & $\begin{array}{l}\text { Indigofera } \\
\text { heterantha } \\
\text { Wall. ex } \\
\text { Brandis } \\
\text { Cytotypes } \\
(\mathbf{x = 8 )} \\
\text { (i) The diploid } \\
\\
\text { (ii) The } \\
\text { hexaploid }\end{array}$ & \begin{tabular}{|l} 
Bhabanagar, \\
1900 \\
Ponda, 1980 \\
\\
Kuppa, 2600 \\
Sangla, 2680 \\
Sangla, 2680 \\
Rakchham, \\
3115 \\
Rakchham, \\
3115 \\
\end{tabular} & $\begin{array}{c}31^{\circ} 33^{\prime} 50^{\prime \prime} \mathrm{N} 77^{\circ} 56^{\prime} 17^{\prime \prime} \mathrm{E} \\
31^{\circ} 33^{\prime} 50^{\prime \prime} \mathrm{N} 77^{\circ} 56^{\prime} 17^{\prime \prime} \mathrm{E} \\
31^{\circ} 25^{\prime} 44^{\prime \prime} \mathrm{N} 78^{\circ} 14^{\prime} 40^{\prime \prime} \mathrm{E} \\
31^{\circ} 25^{\prime} 36^{\prime \prime} \mathrm{N} 78^{\circ} 15^{\prime} 51^{\prime \prime} \mathrm{E} \\
31^{\circ} 22^{\prime} 49^{\prime \prime} \mathrm{N} 78^{\circ} 22^{\prime} 5^{\prime \prime} \mathrm{E} \\
,\end{array}$ & $\begin{array}{l}53718 \\
53719 \\
53722 \\
53720 \\
50911 \\
50912 \\
53723\end{array}$ & $\begin{array}{l}24 \\
24 \\
24 \\
24 \\
24\end{array}$ & $\begin{array}{l}2 x \\
2 x \\
6 x \\
6 x \\
6 x \\
6 x \\
6 x\end{array}$ & $\begin{array}{l}21.11- \\
26.00\end{array}$ & $\begin{array}{l}100 \\
100 \\
\\
100 \\
100 \\
100 \\
98 \\
99\end{array}$ & $2 \mathrm{n}=16,48$ \\
\hline 15. & $\begin{array}{l}\text { I. himalayensis } \\
\text { Ali }\end{array}$ & Kangoos, 1980 & $31^{\circ} 33^{\prime} 9^{\prime \prime} \mathrm{N} 77^{\circ} 55^{\prime} 47^{\prime \prime E}$ & 53717 & 8 & $2 \mathrm{x}$ & - & 100 & $\#$ \\
\hline 16. & $\begin{array}{l}\text { Lotus } \\
\text { corniculatus L. }\end{array}$ & \begin{tabular}{|l|} 
Chittkul, 3450 \\
Moorang, 2590 \\
Thangi, 2700 \\
\end{tabular} & $\begin{array}{c}31^{\circ} 21^{\prime} 7 " \mathrm{~N} 78^{\circ} 26^{\prime} 7 " \mathrm{E} \\
31^{\circ} 35^{\prime} 18^{\prime \prime N} 78^{\circ} 26^{\prime} 53^{\prime \prime} \mathrm{E} \\
31^{\circ} 33^{\prime} 6^{\prime \prime N} 78^{\circ} 28^{\prime} 57^{\prime \prime} \mathrm{E}\end{array}$ & $\begin{array}{l}53690 \\
53691 \\
53692\end{array}$ & $\begin{array}{l}6 \\
6 \\
6\end{array}$ & $\begin{array}{l}2 \mathrm{x} \\
2 \mathrm{x} \\
2 \mathrm{x}\end{array}$ & 18.34 & $\begin{array}{l}88 \\
98 \\
96\end{array}$ & $\begin{array}{l}2 \mathrm{n}=12,24 \\
28,32,36\end{array}$ \\
\hline 17. & $\begin{array}{l}\text { Medicago } \\
\text { falcata } \mathrm{L} \text {. }\end{array}$ & \begin{tabular}{|l|} 
Pooh, 2840 \\
Pooh, 2840 \\
Moorang, 2590 \\
Moorang, 2590 \\
Thangi, 2700 \\
\end{tabular} & $\begin{array}{l}31^{\circ} 45^{\prime} 47^{\prime \prime N} 78^{\circ} 35^{\prime} 20^{\prime \prime E} \\
31^{\circ} 45^{\prime} 47^{\prime \prime N} 78^{\circ} 35^{\prime} 20^{\prime \prime} \mathrm{E} \\
31^{\circ} 35^{\prime} 18^{\prime \prime N} 78^{\circ} 26^{\prime} 53^{\prime \prime} \mathrm{E} \\
31^{\circ} 33^{\prime} 6^{\prime \prime N} 78^{\circ} 28^{\prime} 57^{\prime \prime E}\end{array}$ & $\begin{array}{l}53694 \\
53697 \\
53693 \\
53696 \\
53695\end{array}$ & $\begin{array}{l}8 \\
8 \\
8 \\
8 \\
8\end{array}$ & $\begin{array}{l}2 \mathrm{x} \\
2 \mathrm{x} \\
2 \mathrm{x} \\
2 \mathrm{x} \\
2 \mathrm{x}\end{array}$ & $\begin{array}{l}15.43- \\
52.11\end{array}$ & $\begin{array}{l}90 \\
91 \\
92 \\
92 \\
89\end{array}$ & $2 n=16,32$ \\
\hline 18. & $\begin{array}{l}\text { Melilotus alba } \\
\text { Lamk. }\end{array}$ & $\begin{array}{l}\text { Spello, } 2800 \\
\text { Pooh, } 2840 \\
\text { Reckong Peo, } \\
2670 \\
\text { Thangi, } 2700 \\
\end{array}$ & $\begin{array}{l}31^{\circ} 39^{\prime} 30^{\prime \prime} \mathrm{N} 78^{\circ} 26^{\prime} 28^{\prime \prime} \mathrm{E} \\
31^{\circ} 45^{\prime} 47^{\prime \prime} \mathrm{N} 78^{\circ} 35^{\prime} 20^{\prime \prime} \mathrm{E} \\
31^{\circ} 33^{\prime} 6^{\prime \prime N} 78^{\circ} 16^{\prime} 22^{\prime \prime} \mathrm{E} \\
31^{\circ} 33^{\prime} 6^{\prime \prime N} 78^{\circ} 28^{\prime} 57^{\prime \prime} \mathrm{E}\end{array}$ & $\begin{array}{l}53700 \\
50908 \\
50907 \\
53699\end{array}$ & $\begin{array}{l}8 \\
8 \\
8 \\
8\end{array}$ & $\begin{array}{l}2 \mathrm{x} \\
2 \mathrm{x} \\
2 \mathrm{x} \\
2 \mathrm{x}\end{array}$ & 8.67 & $\begin{array}{l}100 \\
100 \\
95 \\
96\end{array}$ & $\begin{array}{l}2 \mathrm{n}=16,24, \\
32,36\end{array}$ \\
\hline 19. & \begin{tabular}{|l} 
Oxytropis \\
mollis Royle ex \\
Benth. \\
\end{tabular} & \begin{tabular}{|l} 
Rakchham, \\
3115 \\
Chittkul, 3450 \\
\end{tabular} & $\begin{array}{l}31^{\circ} 22^{\prime} 49^{\prime \prime} \mathrm{N} 78^{\circ} 22^{\prime} 5^{\prime \prime} \mathrm{E} \\
31^{\circ} 21^{\prime} 7 " \mathrm{~N} 78^{\circ} 26^{\prime} 7 " \mathrm{E}\end{array}$ & $\begin{array}{l}53702 \\
53701\end{array}$ & $\begin{array}{l}8 \\
8\end{array}$ & $\begin{array}{l}2 \mathrm{x} \\
2 \mathrm{x}\end{array}$ & - & $\begin{array}{l}100 \\
100\end{array}$ & $2 n=32$ \\
\hline 20. & $\begin{array}{l}\text { Piptanthus } \\
\text { nepalensis D. } \\
\text { Don }\end{array}$ & Sangla, 2680 & $31^{\circ} 25^{\prime} 36^{\prime \prime} \mathrm{N} 78^{\circ} 15^{\prime} 51^{\prime \prime E}$ & 53703 & 9 & $2 \mathrm{x}$ & - & 100 & $2 n=18$ \\
\hline 21. & $\begin{array}{l}\text { Trifolium } \\
\text { pratense } \mathrm{L} .\end{array}$ & Sangla, 2680 & $31^{\circ} 25^{\prime} 36^{\prime \prime} \mathrm{N} 78^{\circ} 15^{\prime} 51^{\prime \prime E}$ & 50917 & 7 & $2 \mathrm{x}$ & - & 98 & $\begin{array}{l}2 \mathrm{n}=14,16 \\
26,27,28 \\
29,32,48\end{array}$ \\
\hline 22. & T. repens $\mathrm{L}$. & $\begin{array}{l}\text { Sangla, } 2680 \\
\text { Nichar, } 2150\end{array}$ & $\begin{array}{l}31^{\circ} 25^{\prime} 36^{\prime \prime} \mathrm{N} 78^{\circ} 15^{\prime} 51^{\prime \prime E} \\
31^{\circ} 33^{\prime} 20^{\prime \prime} \mathrm{N} 77^{\circ} 57^{\prime} 21^{\prime \prime E}\end{array}$ & $\begin{array}{l}50916 \\
53711\end{array}$ & $\begin{array}{l}16 \\
16\end{array}$ & $\begin{array}{l}4 \mathrm{x} \\
4 \mathrm{x}\end{array}$ & $5.34-6.02$ & $\begin{array}{l}90 \\
91\end{array}$ & $\begin{array}{l}2 \mathrm{n}=16,22 \\
28,30,32 \\
48,64\end{array}$ \\
\hline 23. & $\begin{array}{l}\text { Trigonella } \\
\text { emodi Benth. }\end{array}$ & $\begin{array}{l}\text { Rakchham, } \\
3115 \\
\text { Chittkul, } 3450 \\
\end{array}$ & $\begin{array}{c}31^{\circ} 22^{\prime} 49^{\prime \prime} \mathrm{N} 78^{\circ} 22^{\prime} 5 " \mathrm{E} \\
31^{\circ} 21^{\prime} 7^{\prime \prime N} 78^{\circ} 26^{\prime} 7 " \mathrm{E}\end{array}$ & $\begin{array}{l}53714 \\
53713\end{array}$ & $\begin{array}{l}8 \\
8\end{array}$ & $\begin{array}{l}2 \mathrm{x} \\
2 \mathrm{x}\end{array}$ & $\begin{array}{l}15.88- \\
17.24\end{array}$ & $\begin{array}{l}94 \\
93\end{array}$ & $2 n=16,24$ \\
\hline 24. & $\begin{array}{l}\text { T. pubescens } \\
\text { Edgew. ex }\end{array}$ & $\begin{array}{l}\text { Rakchham, } \\
3115\end{array}$ & $\begin{array}{l}31^{\circ} 22^{\prime} 49^{\prime \prime N} 78^{\circ} 22^{\prime} 5 " \mathrm{E} \\
31^{\circ} 21^{\prime} 7^{\prime \prime N ~} 78^{\circ} 26^{\prime} 7 " \mathrm{E}\end{array}$ & $\begin{array}{l}53716 \\
53715\end{array}$ & $\begin{array}{l}8 \\
8\end{array}$ & $\begin{array}{l}2 \mathrm{x} \\
2 \mathrm{x}\end{array}$ & $\begin{array}{l}47.05- \\
62.88\end{array}$ & $\begin{array}{l}38 \\
54\end{array}$ & $\#$ \\
\hline
\end{tabular}

Volume 5 Issue 5, May 2016 www.ijsr.net 
International Journal of Science and Research (IJSR)

ISSN (Online): 2319-7064

Index Copernicus Value (2013): 6.14 | Impact Factor (2015): 6.391

\begin{tabular}{|c|c|c|c|c|c|c|c|c|c|}
\hline & Baker & Chittkul, 3450 & & & & & & & \\
\hline 25. & $\begin{array}{l}\text { Vicia pallida } \\
\text { Turcz. } \\
\text { (=V. sylvatica } \\
\text { Benth.) }\end{array}$ & $\begin{array}{l}\text { Chaura, } 1890 \\
\text { Bhabanagar, } \\
1900 \\
\text { Palingi, } 1900\end{array}$ & $\begin{array}{l}31^{\circ} 33^{\prime} 50^{\prime \prime} \mathrm{N} 77^{\circ} 56^{\prime} 17^{\prime \prime} \mathrm{E} \\
31^{\circ} 33^{\prime} 50^{\prime \prime} \mathrm{N} 77^{\circ} 567^{\prime} 17^{\prime \prime} \\
31^{\circ} 33^{\prime} 23^{\prime \prime N} 77^{\circ} 52^{\prime} 58^{\prime \prime} \mathrm{E}\end{array}$ & $\begin{array}{l}53707 \\
53706 \\
53708\end{array}$ & $\begin{array}{l}12 \\
12 \\
12\end{array}$ & $\begin{array}{l}4 x \\
4 x \\
4 x\end{array}$ & $1.52-2.20$ & $\begin{array}{l}99 \\
73 \\
79\end{array}$ & $2 n=14$ \\
\hline 26. & $\begin{array}{l}V . \text { rigidula } \\
\text { Royle }\end{array}$ & $\begin{array}{l}\text { Kalpa, } 2760 \\
\text { Kalpa, } 2760\end{array}$ & $\begin{array}{l}31^{\circ} 31^{\prime} 55^{\prime \prime} \mathrm{N} 78^{\circ} 15^{\prime} 5^{\prime \prime} \mathrm{E} \\
31^{\circ} 31^{\prime} 55^{\prime \prime} \mathrm{N} 78^{\circ} 15^{\prime} 5^{\prime \prime} \mathrm{E}\end{array}$ & $\begin{array}{l}50919 \\
51044\end{array}$ & $\begin{array}{l}12 \\
12\end{array}$ & $\begin{array}{l}4 x \\
4 x\end{array}$ & 6.52 & $\begin{array}{l}80 \\
81\end{array}$ & $\#$ \\
\hline 27. & $V$. sativa $\mathrm{L}$. & $\begin{array}{l}\text { Reckong Peo, } \\
2670 \\
\text { Kuppa, } 2600\end{array}$ & $\begin{array}{c}31^{\circ} 33^{\prime} 6^{\prime \prime} \mathrm{N} 78^{\circ} 16^{\prime} 22^{\prime \prime} \mathrm{E} \\
31^{\circ} 25^{\prime} 44^{\prime \prime} \mathrm{N} 78^{\circ} 14^{\prime} 40^{\prime \prime} \mathrm{E}\end{array}$ & $\begin{array}{l}50918 \\
53709\end{array}$ & $\begin{array}{l}6 \\
6\end{array}$ & $\begin{array}{l}2 x \\
2 x\end{array}$ & - & $\begin{array}{l}100 \\
100\end{array}$ & $\begin{array}{l}2 \mathrm{n}=12,14, \\
24\end{array}$ \\
\hline 28. & V. tenera Grah. & $\begin{array}{l}\text { Kuppa, 2600 } \\
\text { Sangla, 2680 } \\
\text { Sangla, 2680 } \\
\text { Sangla, 2680 } \\
\text { Sangla, 2680 }\end{array}$ & $\begin{array}{c}31^{\circ} 25^{\prime} 44^{\prime \prime} \mathrm{N} 78^{\circ} 14^{\prime} 40^{\prime \prime} \mathrm{E} \\
31^{\circ} 25^{\prime} 36^{\prime \prime} \mathrm{N} 78^{\circ} 15^{\prime} 51^{\prime \prime} \mathrm{E} \\
, \\
, \\
,\end{array}$ & $\begin{array}{l}53710 \\
50920 \\
51950 \\
51951 \\
51952\end{array}$ & $\begin{array}{l}7 \\
7 \\
7 \\
7 \\
7\end{array}$ & $\begin{array}{l}2 x \\
2 x \\
2 x \\
2 x \\
2 x\end{array}$ & - & $\begin{array}{c}100 \\
94 \\
93 \\
94 \\
93\end{array}$ & \# \\
\hline
\end{tabular}

* Code of Herbarium maintained by the Department of Botany, Punjabi University, Patiala, India as per "Index Herbariorum" by Holmgren and Holmgren (1998).

**Darlington and Wylie (1955), Fedorov (1969), Moore (1967-1971, 1968, 1969, 1970, 1972 1973-1974), Löve and Löve (1982a,b, 1986), Goldblatt (1975-1978, 1979-1981, 1982-1983, 1984-1985), Goldblatt and Johnson (1986-1987, 1988-1989, 1990-1991, 1992-1993, 1994-1995, 1996-1997, 1998-2000, 2001-2003), Kumar and Subramanian (1986), Khatoon and Ali (1993) and IAPT/IOPB Chromosome Number Reports published in Taxon and Index to Plant Chromosome Numbers (wide internet site: http://mobot.mobot.org/W3T/Search/ipen.html and www.tropicos.org/Project/IPCN) and other journals.

${ }^{\text {\# }}$ First ever chromosome count in the species. cytotype of $2 \mathrm{n}=32$ as recorded by Ashraf and Gohil, 1986 [1] from Kashmir Himalayas. For Trigonella emodi $(2 \mathrm{n}=16$, 2x) (Fig. 15), Sareen and Singh (1976) [11] earlier recorded the triploid cytotype with $2 \mathrm{n}=24$ from Indian North West Himalayas. In Vicia pallida, a new tetraploid cytotype with a chromosome count of $2 n=24$ (Fig. 16) is added against the earlier diploid chromosome reports of $2 n=14$ (based on $\mathrm{x}=6$ ) recorded from Kumaon Hills in Western Himalayas [6].

\subsection{Intraspecific polyploid cytotypes}

Presently, in Indigofera heterantha existence of intraspecific diploid ( $\mathrm{n}=8$; Fig. 17) and hexaploid ( $\mathrm{n}=24$; Fig. 18) cytotypes (based on $\mathrm{x}=8$ ) are reported. The values for morphological characters, stomatal size and index were found to be more in the hexaploid individuals compared to the diploid individuals (see: Table 2).

Table 2: Comparison of macro- and microscopic morphological characters of the diploid and hexaploid cytotypes of

\begin{tabular}{|l|l|l|}
\hline \multicolumn{3}{|c|}{ Indigofera heterantha Wall. ex Brandis } \\
\hline Characters & \multicolumn{2}{c|}{ Cytotypes } \\
\hline Plant height $(\mathrm{cm})$ & \multicolumn{1}{|c|}{ Diploid $(\mathrm{n}=8)$} & \multicolumn{1}{c|}{ Hexaploid $(\mathrm{n}=24)$} \\
\hline No. of branches per plant & $30.12-40.68$ & $58.88-99.24$ \\
\hline No. of leaflets per leaf & Less branched & More branched \\
\hline Size of leaf $(\mathrm{cm})$ & $7-13$ & $19-23$ \\
\hline Size of leaflet $(\mathrm{cm})$ & $2.50-5.50$ & $6.00-10.50$ \\
\hline Colour of leaves & $0.40-1.00 \times 0.10-0.60$ & $0.50-1.60 \times 0.20-1.00$ \\
\hline No. of flowers per inflorescence & Light green & Dark green \\
\hline Colour of flowers & Dark purple & $12-21$ \\
\hline Stomatal size $(\mu \mathrm{m})$ & $22.62-24.50 \times 21.86-23.37$ & Purple \\
\hline Stomatal index & 16.67 & $26.39-27.14 \times 25.63-26.39$ \\
\hline
\end{tabular}

\subsection{Meiosis, microsporogenesis, and pollen fertility}

Meiotic course in majority of species which include chromosomal pairing, bivalents formation, segregation of chromosomes during A-I/T-I, A-II/T-II was observed to be perfectly regular resulting into normal sporads and high pollen fertility. However, 11 species viz., Astragalus grahamianus, A. graveolens, Indigofera heterantha, Lotus corniculatus, Medicago falcata, Melilotus alba, Trifolium repens, Trigonella emodi, T. pubescens, Vicia pallida, and $V$. rigidula depict the phenomenon of cytomixis involving chromatin transfer among neighboring PMCs (Figs.19-20). Consequent to chromatin transfer, the PMCs involved in cytomixis depict various meiotic irregularities which includes hypo-hyperploid PMCs (Fig. 21), enucleated PMCs (Fig. 22), chromatin stickiness (Fig. 23), interbivalents connections, out of plate bivalents and laggards (Fig. 24) due to spindle irregularities, sporads with micronuclei (Fig. 25) and polyads (Fig. 26). The products of such sporads yielded unstained/sterile and fertile pollen grains of variable sizes (Figs. 27-28). Such an impact of cytomixis in inducing meiotic irregularities and pollen malformation has also been detected earlier in Vicia faba [4], Clematis graveolens [12], Dianthus angulatus [5], and Lindelofia longiflora var. falconeri [8]. 


\section{International Journal of Science and Research (IJSR) \\ ISSN (Online): 2319-7064}

Index Copernicus Value (2013): 6.14 | Impact Factor (2015): 6.391

\section{References}

[1] Ashraf, M., Gohil, R.N. 1986. Chromosome number reports LXXXXI. Taxon 35: 408.

[2] Baquar, S.R., Abid Askari, S.H. 1970. Chromosome numbers in some flowering plants of west Pakistan. Genet. Iber. 22: 1-11.

[3] Chauhan, N.S. 2003. Important medicinal and aromatic plants of Himachal Pradesh. Indian Forester 129: 979998.

[4] Haroun, S.A., Al Shehri, A.M., Al Wadie, H.M. 2004. Cytomixis in the microsporogenesis of Vicia faba $\mathrm{L}$. (Fabaceae). Cytologia 69: 7-11.

[5] Kumar, P., Singhal, V.K., Kaur, D. 2012. Impaired male meiosis due to irregular synapsis coupled with cytomixis in a new diploid cytotype of Dianthus angulatus (Caryophyllaceae) from Indian cold deserts. Folia Geobot. 47: 59-68. doi: 10.1007/s12224-0119107-8

[6] Mehra, P.N., Dhawan, H. 1971. In IOPB chromosome number reports XXXIV Taxon 20: 785-797.

[7] Mabberley, D.J. 1997. The plant book, 2nd ed. Cambridge University Press, Cambridge, UK.
[8] Rana, P.K., Kumar, P., Singhal V.K. 2014 Cytomixis and associated abnormalities during male meiosis in Lindelofia longiflo ra var. falconeri (Boraginaceae) Cytologia 79(4): 535540.

[9] Rawat, D.S., Kharwal, A.D. 2011. Traditional health practices by 'kinners'- a tribe in alpine and sub-alpine Himalayas of Kinnaur (Himachal Pradesh), India. Life sciences Leaflets 22: 1048-1055.

[10] Rundel, R.W. 1989. Ecological success in relation to plant form and function in the woody legumes. In C. H. Stirton and J. L. Zarucchi [eds.], Advances in legume biology, Monographs in Systematic Botany from the Missouri Botanical Garden 29: 377-398.

[11] Sareen, T.S., Singh, P.D. 1976. Cytological studies in some north Indian Leguminosae. Proc. Indian Sci. Congr. Assoc. 93: 122-123.

[12] Singhal, V.K., Kaur, D. 2011. Cytomixis inducing meiotic irregularities and pollen malformation in Clematis graveolens Lindley from the cold deserts of Kinnaur districts of Himachal Pradesh, India. Cytologia 76: 319-327.

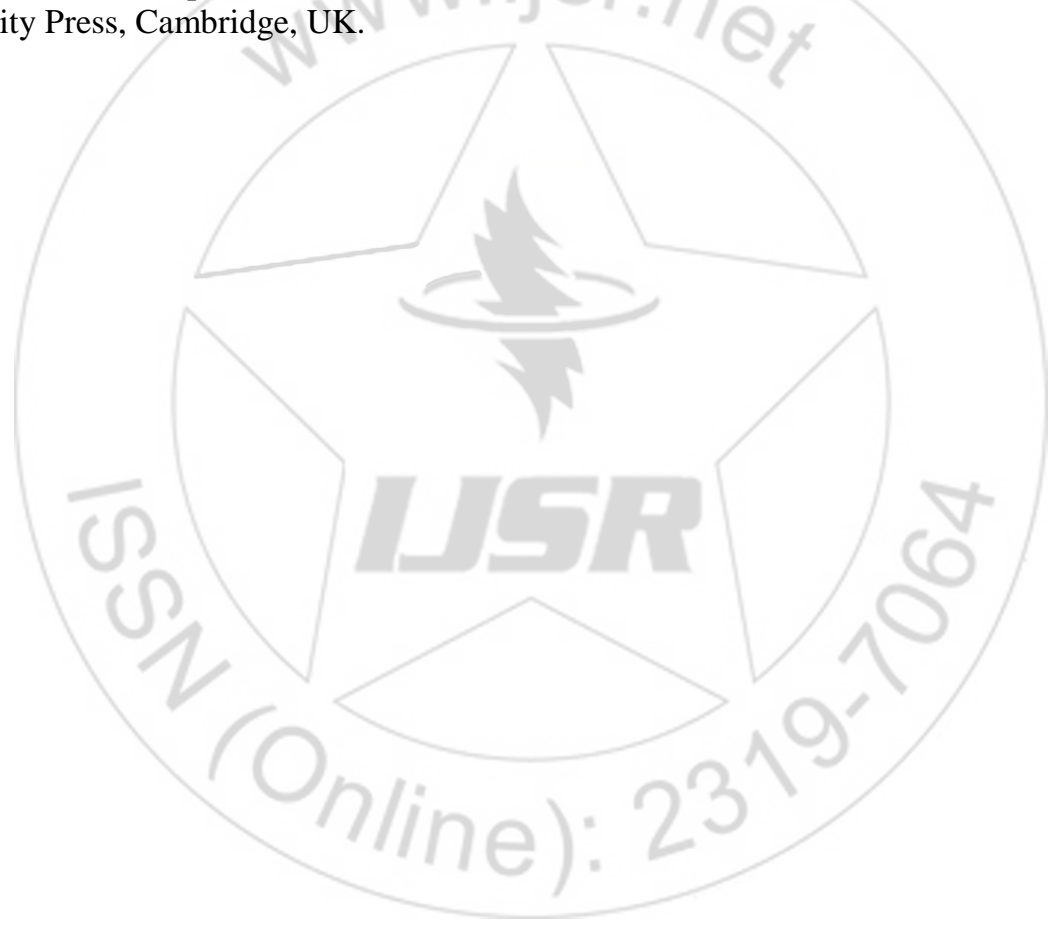




\section{International Journal of Science and Research (IJSR) \\ ISSN (Online): 2319-7064}

Index Copernicus Value (2013): 6.14 | Impact Factor (2015): 6.391

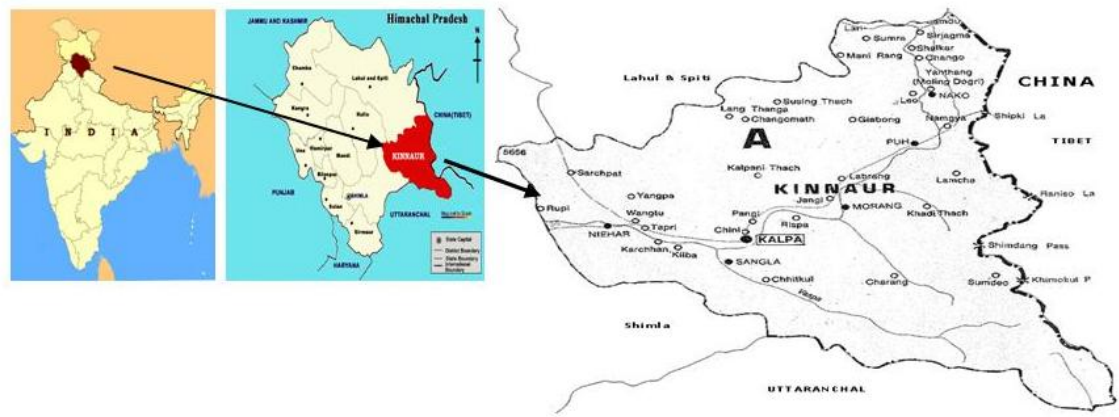

Map 1 : The map showing various localities of Kinnaur district of Himachal Pradesh in India.
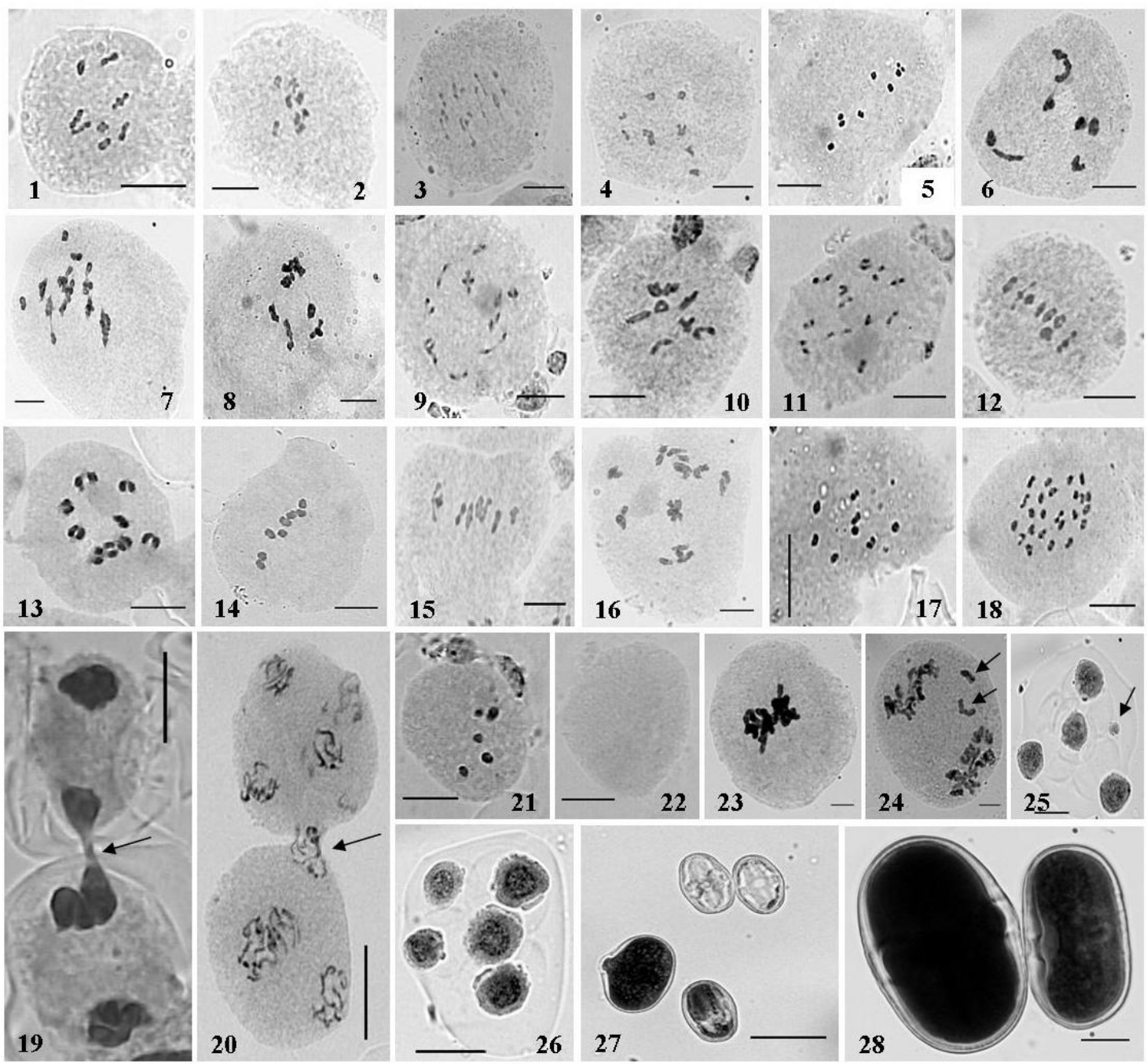

12
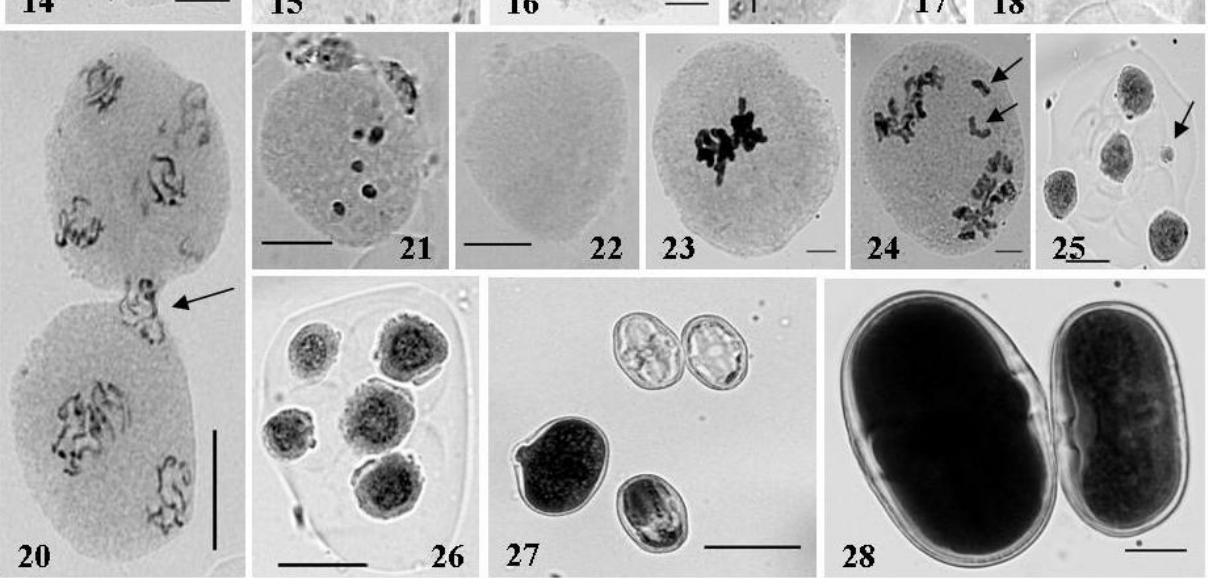

Legends of figures

Fig. 1) Astragalus malacophyllus, A PMC with $8_{\mathrm{II}}$ at M-I. 2) A strobiliferus, A PMC with $8_{\mathrm{II}}$ at M-I. 3) Campylotropis eriocarpa, A PMC with $11_{\text {II }}$ at M-I. 4) Colutea nepalensis, A PMC with $8_{\text {II }}$ at M-I. 5) Indigofera himalayensis, A PMC with $8_{\text {II }}$ at M-I. 6) Trigonella pubescens, A PMC with $8_{\mathrm{II}}$ at diakinesis. 7) Vicia rigidula, A PMC with $12_{\mathrm{II}}$ at M-I. 8) V. tenera, A PMC with $7_{\mathrm{II}}$ at MI. 9) Desmodium elegans var. nutans, A PMC with $11_{\text {II }}$ at diakinesis. 10) Caragana gerardiana, A PMC with $8_{\text {II }}$ at M-I. 11) Desmodium racemosum, A PMC with $11_{\text {II }}$ at diakinesis. 12) Medicago falcata, A PMC with $8_{\text {II }}$ at M-I. 13) Piptanthus nepalensis, A PMC with $9_{\text {II }}$ at diakinesis. 14) Oxytropis mollis, A PMC with $8_{\mathrm{II}}$ at M-I. 15) Trigonella emodi, A PMC with $8_{\mathrm{II}}$ at M-I. 16) Vicia pallida, A PMC with $12_{\mathrm{II}}$ at diakinesis. 17) Indigofera heterantha, A PMC with $8_{\text {II }}$ at M-I. 18) Indigofera heterantha, A PMC with $24_{\text {II }}$ at M-I. 19) Two PMCs involved in chromatin transfer (arrowed) at A-I. 20) Two PMCs involved in chromatin transfer (arrowed) at A-II. 21) A hypoploid PMC. 22) A enucleated PMC. 23) A PMC at M-I showing chromatin stickiness. 24) A PMC at A-I showing laggards (arrowed). 25) A tetrad with one micronucleus (arrowed). 26) A polyad. 27) Sterile pollen grains. 28) Apparently fertile heterogeneous sized pollen grains. 